\title{
EFFICACY OF GLYCERYL TRINITRATE OINTMENT FOR PERIANAL- INTRARECTAL LOCALANESTHESIA IN TRANSRECTAL ULTRASOUND- GUIDED PROSTATE BIOPSY- COMPARED TO TOPICAL LIGNOCAINE
}

\author{
SK. AMIRUL ISLAM ${ }^{1}$, AKM KHURSHIDUL ALAM ${ }^{2}$, MOHAMMAD SHAFIQUR RAHMAN ${ }^{2}$, MD. MONOWARUL \\ ISLAM ${ }^{1}$, MOHAMMED MIZANUR RAHMAN ${ }^{3}$, PRANASHIS SAHA ${ }^{1}$
}

${ }^{1}$ Department of Uro-Oncology, National Institute of Cancer research \& Hospital (NICRH), Mohakhali, Dhaka, ${ }^{2}$ Department of Urology, Bangabandhu Sheikh Mujib Medical University, Shahbagh, Dhaka, ${ }^{3}$ Deputy Director, Central Medical Store Depot, Dhaka

\begin{abstract}
Objective: Anorectal pain and discomfort during transrectal ultrasound (TRUS)-guided prostate biopsy is an important issue of this procedure. Therefore present study was designed to compare whether the use of topical glyceryl trinitrate (GTN) ointment or lignocaine gel is more effective as an adjunct to periprostatic nerve block (PPNB) in reducing pain associated with transrectal ultrasound (TRUS)-guided prostate biopsy.

Methods: In all, 120 consecutive patients having their first TRUS-guided prostate biopsy were randomized to receive either $0.4 \%$ GTN ointment or $2 \%$ lignocaine gel, 10 minutes or 5 minutes respectively before biopsy. All patients had a biopsy preceded by an injection with $5 \mathrm{ml}$ of $2 \%$ lignocaine local anaesthesia.

Results: A 100-point visual analogue score was used to record overall pain due to the presence of the probe, the biopsy itself and pain after the procedure. There was no significant difference in age, PSA level and prostate volume between the groups. There was a significantly lower mean pain score due to probe insertion, in the GTN than lignocaine group (18.32 vs 37.48, $P<0.001)$; pain perception was lower for the whole procedure in the GTN group (16.59 vs 35.17, $P<0.001$ ); and was most pronounced in men aged d"65 years $(21.45$ vs $40.7, P<0.001)$. Five patients $(8.3 \%)$ in the GTN group complained of headache.

Conclusion: So it can be concluded that, topical GTN ointment is safe and effective in reducing the discomfort and pain associated with TRUS-guided prostate biopsy, in particular the insertion of the ultrasound probe. It might be of maximum benefit in the younger patient(d"65years).
\end{abstract}

Key words: Glyceryl trinitrate, Transrectal ultrasound, Prostate biopsy, Analgesia

Bangladesh J. Urol. 2018; 21(1): 14-19

\section{Introduction}

Prostate cancer is the most common cancer in men. In England and Wales, the incidence of prostate cancer has been increasing steadily and has more than doubled in the last two decades. Although screening tests such as the digital rectal examination (DRE), transrectal ultrasound(TRUS) and prostate specific antigen (PSA)

Correspondence: Dr. Sk. Amirul Islam, Dept. of Uro-Oncology, NICRH, Mohakhali, Dhaka, Email: amiruluro@yahoo.com assay help in prostate cancer detection, their lack of specificity and sensitivity confound the diagnosis ${ }^{1}$.

During the '80s, TRUS guided prostate biopsy became the primary technique for the detection of prostate cancer. Because cancers cannot be accurately visualized by conventional ultrasound, sextant biopsy was pioneered by Hodge et al. Over the past decade, a significant number of modifications have been made to the techniques of prostate cancer biopsy ${ }^{2,3}$. Much has 
been written about the indications for biopsy, the patient preparation, the potential complications associated with the procedure and the optimal number of biopsy cores 4 . Studies have shown that an extended biopsy scheme consisting of 10-12 biopsies from the prostate gland maximizes the detection rate of prostate cancer. Thus, these protocols have become the norm in the diagnosis of prostate cancer ${ }^{5,6}$.

In clinical practice, patient preparation for transrectal prostate biopsy has three basic steps: rectal preparation, antibiotic prophylaxis, and analgesia ${ }^{3}$. Minor emphasis has been placed on the problem of pain during the procedure since patient discomfort has usually been considered a necessary part of it. In 2000 Soloway and Obek from the University of Miami strongly stressed for the first time the importance of analgesia/ anesthesia as a necessary part of the biopsy procedure ${ }^{4,7}$. Pain during TRUS guided prostate biopsy has a 2-fold source, originating from the insertion of the TRUS probe in the rectum, which is innervated below the dentate line by the inferior rectal branches of the pudendal nerve, and from multiple needle punctures through the prostate capsule, which is innervated by autonomic branches of the neurovascular bundles running between the posterolateral aspect of the prostate and rectum ${ }^{\mathbf{8 , 9}}$.

Several techniques such as periprostatic nerve blockage, rectal administration of lignocaine gel, intravenous propofol, inhalation of nitrous oxide, and the use of tramadol have been described in the literature for adequate pain control during biopsy. The discomfort during the prostatic biopsy is proportional to the number of cores ${ }^{10}$. Although periprostatic nerve block (PPNB) is currently considered the gold standard for pain control during prostate biopsy (PBx), it does not eliminate probe related anorectal discomfort and it may even add considerable pain due to the transcapsular infiltration of local anesthesia ${ }^{9}$. To reduce probe related pain and discomfort topical lignocaine gel has been used for many years. It is widely accepted that the most effective pain relief is probably achieved by combining the anorectal application of a topical anesthetic and periprostatic nerve block ${ }^{3}$.

Topical glyceryl trinitrate (GTN) ointment has been used widely for the management of chronic anal fissure. GTN has been shown to decrease the anal tone and release nitric oxide that is identified as mediating relaxation of the anal sphincter. GTN application to the anus causes a fall in maximum anal resting pressure within 10 minutes, lasting for up to 90 minutes after application.
Some studies reported the effectiveness and efficacy of the GTN ointment in prostate biopsy ${ }^{6}$. So topical GTN ointment is safe and effective in reducing the discomfort associated with TRUS-guided biopsy of the prostate, in particular the insertion of the ultrasound probe ${ }^{11,12}$.

Prostate cancer is not uncommon in Bangladesh. Therefore, this study was proposed to compare the efficacy of glyceryl trinitrate ointment over lignocaine gel in reducing anal pain and discomfort during transrectal ultrasound guided prostate biopsy. This will help in minimizing the pain and discomfort associated with transrectal ultrasound guided prostate biopsy more effectively in future.

\section{Patients \& Methods}

This single blind randomized controlled trial was conducted in the Department of Urology, Bangabandhu Sheikh Mujib Medical University (BSMMU) from March, 2011 to February, 2012 after receiving approval from Institutional Review Board(IRB) to compare whether the perianal- intrarectal glyceryl trinitrate ointment or $2 \%$ lignocaine gel is more effective in reducing anal discomfort and pain during transrectal ultrasound guided prostate biopsy.

Patients less than 75 years of age with raised serum prostate-specific antigen (PSA), abnormal digital rectal examination (DRE), or both were enrolled in this study. Exclusion criteria were coagulation disorders, hemorrhoidal disease, severe cardiac or hepatic disease, those on nitrate medication or recent phosphodiesterase-5 inhibitor use. Patients with previous prostate biopsies were also excluded from this study. A total of 120 patients were scheduled to undergo TRUSguided prostate biopsy according to the selection criteria and placed into two groups by random allocation on lottery basis for this study. Selected patient was evaluated preoperatively by history, physical examination and relevant investigation. All patients received bowel preparation and oral antibiotic prophylaxis (Ciprofloxacin $500 \mathrm{mg}$ and Metroidazole $400 \mathrm{mg}$ ) the day before the procedure and antibiotic was continued for 5 days .

An intravenous infusion was started before the procedure. Patient was placed in the left lateral(sims) position. The experimental group received $1 \mathrm{gm}$ of $0.4 \%$ glyceryl trinitrate $(4 \mathrm{mg})$ ointment whereas the control group received $2 \%$ lignocaine gel both into the anal canal and perianal area. After 10 and 5 minutes respectively ultrasound probe (7.5 MHz TRUS probe) was inserted per rectally and prostate volume was 
calculated. Periprostatic nerve block (PPNB) was given by $5 \mathrm{ml}$ lignocaine $(2 \%)$ solution under ultrasound guidance using biopsy needle. Biopsies was taken immediately after application of PPNB using a springloaded biopty gun with an 18- G needle. All patients were underwent 12 core biopsies scheme protocol as the standard procedure.

For evaluation, Visual Analogue Scale was explained to the patient before the procedure. Then the patients were asked to assess the pain during ultrasound probe insertion, during biopsy procedure and pain after the procedure, with a score range from 0-100 each, with 0 corresponding to no pain, no discomfort and 100 corresponding to maximum pain and discomfort. Total duration of the procedure were recorded. Any additional analgesic requirement, complications during and after the procedure such as; vasovagal reaction, haematuria, per rectal bleeding, haemospermia, fever, urosepsis and headache, and hospital stay more than 4 hours if required were also recorded in the pre-designed data collection sheet. Apart from those patients whose required hospital stay, all other patients were discharged from the post procedure observation room 1 hour after the procedure and adviced to consult (physically or over telephone) if any complication develops after discharge.

After compilation, data were processed and analyzed using the computer based Statistical Package for Social
Sciences (SPSS) software. Results were described as mean \pm standard deviation (SD) and compared by Student's t-test for quantitative data and chi-square (Ç $\left.{ }^{2}\right)$ test for qualitative data. A ' $p$ ' value of $<0.05$ was considered statistically significant.

\section{Results}

One-hundred twenty(120) consecutive TRUS-guided biopsies of the prostate with 12 core scheme were performed. There was no statistical difference in age, indication of biopsy, prostate volume and biopsy time between the 2 groups. Patients' characteristics are summarized in Table 1.

Table 2 lists VAS scores from each group during probe insertion, during biopsy and pain after the procedure. Patients from experimental group who received perianalintrarectal GTN ointment before PPNB, reported significantly less pain and discomfort during all three times of measurement compared with control group who treated with topical lignocaone gel before PPNB. The difference in VAS score between the two groups were statistically highly significant during all three times of measurement $(p<0.001)$.

In table 3 correlations of age, prostate volume and duration of biopsy with mean pain score were analyzed. Only age of the patient seems to have a significant correlation with the pain scores $(p<0.01)$, being significantly lower in patients age $>65$ years which is shown in table 4.

Table I

Patient demographic and baseline characteristics for the study groups.

\begin{tabular}{|c|c|c|c|}
\hline Variables & $\begin{array}{l}\text { Experimental group } \\
\qquad(\mathrm{n}=60)\end{array}$ & $\begin{array}{l}\text { Control group } \\
(n=60)\end{array}$ & p-value \\
\hline \multicolumn{4}{|l|}{ Age (years) } \\
\hline Mean \pm SD & $65.55 \pm 6.32$ & $66.17 \pm 6.18$ & $0.674^{\mathrm{ns}}$ \\
\hline Range & $48-75$ & $51-75$ & \\
\hline \multicolumn{4}{|l|}{ Indication of biopsy } \\
\hline Abnormal DRE & $17(28.3 \%)$ & $12(20 \%)$ & $0.892^{\mathrm{ns}}$ \\
\hline Raised serum PSA level & $29(48.3 \%)$ & $38(63.3 \%)$ & \\
\hline Both & $14(23.3 \%)$ & $10(16.6 \%)$ & \\
\hline \multicolumn{4}{|l|}{ Volume of prostate(gram) } \\
\hline Mean \pm SD & $55.42 \pm 8.4$ & $50.98 \pm 8.65$ & $0.742^{\text {ns }}$ \\
\hline Range & $37-74$ & $34-71$ & \\
\hline \multicolumn{4}{|l|}{ Duration of Procedure(min.) } \\
\hline Mean \pm SD & $19.93 \pm 3.35$ & $24.73 \pm 3.72$ & $0.466^{\mathrm{ns}}$ \\
\hline Range & $13-28$ & $15-36$ & \\
\hline
\end{tabular}

ns $=$ not significant 
Table-II Mean Visual Analogue Scale (VAS) scores for the study groups.

\begin{tabular}{lccc}
\hline VAS Score & $\begin{array}{c}\text { Experimental group } \\
(\mathrm{n}=60)\end{array}$ & $\begin{array}{c}\text { Control group } \\
(\mathrm{n}=60)\end{array}$ & p-value \\
$($ mean \pm SD $)$ & $18.32 \pm 4.86$ & $37.48 \pm 6.19$ & $<0.001^{*}$ \\
\hline During insertion of TRUS probe & $16.78 \pm 4.74$ & $34.95 \pm 6.10$ & $<0.001^{*}$ \\
During biopsy & $14.68 \pm 4.59$ & $33.12 \pm 5.77$ & $<0.001^{*}$ \\
After biopsy & & & \\
\hline
\end{tabular}

* = highly significant

Table-III Correlation between mean VAS score and baseline variables.

Variables

Age

Volume of prostate

Duration of biopsy
Correlation coefficient $(r)$

$-0.409$

$-0.034$

0.087

* $=$ significant correlation, $\mathrm{ns}=$ not significant p-value

$<0.01^{*}$

$>0.05^{\mathrm{ns}}$

$>0.05^{\mathrm{ns}}$

Table IV : Comparison of the pain scores according to age of the patient.

\begin{tabular}{lccccc}
\hline & $\leq 65$ years & $>65$ years & 't' & df & p-value \\
\hline Experimental group & & & & & \\
No of cases(n) & 27 & 33 & & & \\
Mean overall VAS \pm & $21.45 \pm 1.34$ & $12.61 \pm 1.78$ & 21.23 & 58 & $<0.001^{*}$ \\
SD & & & & & \\
Control group & 31 & 29 & & & \\
No of cases(n) & $40.70 \pm 1.81$ & $29.26 \pm 1.49$ & 26.56 & 58 & $<0.001^{*}$ \\
Mean overall VAS $\pm S D$
\end{tabular}

${ }^{*}=$ highly significant

Additional analgesia (parenteral/oral) during or after the procedure was required to relieve the discomfort and pain and complete the procedure, for $9(15 \%)$ patients in experimental group and $27(45 \%)$ patients in control group, the difference was statistically significant ( $p$ $<0.001)$.

Twenty five patients $(41.6 \%)$ developed complications during or following biopsy with one $(1.6 \%)$ patient required hospital stay more than 4 hours in experimental group unlike control group in which $23(38.3 \%)$ patients developed complications and 2 (3.3\%) patient required hospital stay. The complications in experimental group were; haematuria in 14 , per rectal bleeding in 2 , fever in 2 and haemospermia in 2 patient. In control group, haematuria in 11, per rectal bleeding in 5 , fever in 3 and haemospermia in 4 patient, but no statistical difference was found among the groups. No patient from any group

\section{(7)}

developed urosepsis. Fever was subsided with continuation of antibiotic for 3 weeks. Other complications were resolved spontaneously. In experimental group, $5(8.3 \%)$ patient developed headache probably due to glyceryl trinitrate administration which was treated by simple analgesic.

\section{Discussion}

Currently, transrectal ultrasound(TRUS) guided prostate biopsy have been widely used for the diagnosis of prostate cancer. Although it is a common procedure usually performed on an outpatient basis, a significant number of patients find the procedure uncomfortable ${ }^{7}$. After the introduction of periprostatic nerve block (PPNB) by Nash and colleagues, several studies reported the necessity of topical anesthesia during prostate biopsy 13. Numerous forms of anesthesia, such as lignocaine gel, intravenous sedation and PPNB have been 
investigated and are widely used ${ }^{14,15,16}$. In addition, although PPNB may prevent the pain that arises from the prostatic capsule or stroma, it seems that it is not useful in preventing the pain arising from probe insertion, especially in young patients.

Topical lignocaine gel has been used in many studies as a local anesthetic for prostate biopsy. It has been also used before periprostatic nerve block (PPNB) anesthesia as a topical perianal anesthetic to decrease anal discomfort caused by probe insertion as well as to improve tolerance ${ }^{17}$. In another study from Philip et al (2006), the authors concluded that the introduction of the transrectal ultrasound probe was significantly more painful than the biopsy after the application of PPNB anesthesia and suggested that the use of a topical perianal anesthetic/ muscle relaxant might be useful ${ }^{1}$.

Topical glyceryl trinitrate (GTN) ointment has been used widely for the management of chronic anal fissure. GTN has been shown to decrease the anal tone and release nitric oxide that is identified as mediating relaxation of the anal sphincter ${ }^{18}$. Previous studies reported the effectiveness and efficacy of the GTN ointment in prostate biopsy ${ }^{11,12}$.

In this study, there was no significant difference in the demographic and baseline variables between the groups. Patient reported pain score was the primary outcome variable in this study. So after TRUS-guided prostate biopsy, the patients were asked to record the pain they experienced during ultrasound probe insertion, during biopsy and pain after the procedure in an unmarked linear visual analogue scale (VAS) between the limits of 0 to 100,0 being no pain at all and 100 being the worst pain possible. The VAS is validated in numerous studies as a reliable tool for assessment of severity of pain. The VAS score is the distance, in millimeters, from the 0 end of the scale to that point marked by the patient as representative of their perceived pain. In our study, patients from experimental group, reported significantly less pain and discomfort during all three times of measurement compared with control group. The difference in VAS score between the two groups were statistically highly significant during all three times of measurement.

In a similar study by Skriapas et al (2011), to study the efficacy of perianal-intrarectal topical GTN ointment on pain associated with TRUS-guided prostate biopsy, they found that pain scores were significantly lower when glyceryl trinitrate (GTN) ointment was used than when lignocaine gel was used, while inserting TRUS probe into the rectum and during biopsy ${ }^{6}$.

In this study, only age of the patient seems to have a significant correlation with the pain scores, being significantly lower in patients age $>65$ years. Skriapas et al (2011), found that pain perception was significantly increased in patients age $<65$ years. This may be related to increased anal tone in young patients. In another study by McCabe et al (2007) reported higher overall pain scores in patients age $<60$ years ( 2.13 vs 4.61 , $p<0.005)^{12}$. Previous studies showed that younger men ( $<60 / 65$ years) have more pain during TRUS guided prostate biopsy and it might be that topical GTN has a role as an adjunct to PPNB in this group. GTN causes smooth muscle relaxation and has been shown to decrease anal sphincter tone, and hence its therapeutic application for treating anal fissure. The evidence from the present study is that GTN offers a significant benefit in reducing the pain from probe insertion, and this then results in the patient's perception of pain throughout the procedure being reduced.

In this study, additional analgesic (parenteral/oral) requirement was higher in control group than experimental group and the difference was statistically significant. Rochester et al (2005), found that $30 \%$ of their control group and $24 \%$ of patients in the glyceryl trinitrate (GTN) group would request additional analgesia if they required repeat biopsy ${ }^{11}$.

In experimental group, 5 (8.3\%) patient developed headache probably due to glyceryl trinitrate administration which was treated by simple analgesic. Skriapas et al (2011), McCabe et al (2007) and Rochester et al (2005) were reported incidence of headache in their study as $11.8 \%, 10 \%$ and $9.7 \%$ respectively due to glyceryl trinitrate administration $6,11,12$. The differences in the overall complication rate and the subsequent hospital stay between the two groups were not statistically significant.

\section{Conclusions}

The findings of this study suggest that perianal-intrarectal topical glyceryl trinitrate ointment is safe and effective in reducing the discomfort and pain associated with transrectal ultrasound guided prostate biopsy, in particular the insertion of the ultrasound probe specially in younger patients (d"65 years) due to increased anal tone, in these group of patient. Except headache in very few patients, no glyceryl trinitrate specific side effect was noted in this study. This study also proves that 
topical $2 \%$ lignocaine gel before periprostatic nerve block (PPNB) does not adequately reduce the discomfort and pain associated with transrectal ultrasound guided prostate biopsy. Hence, use of topical glyceryl trinitrate ointment for transrectal ultrasound guided prostate biopsy is strongly suggested in selected patients in routine clinical practice to increase the tolerance of the procedure.

\section{References}

1. Philip J, McCabe JE, Roy SD, et al. Site of local anaesthesia in transrectal Ultrasonography-guided 12-core prostate biopsy: does it make a difference? BJU Int. 2006 ; 97: 263-265.

2. Hodge KK, McNeal JE, Terris MK, et al. Random systematic versus directed ultrasound guided transrectal core biopsies of the prostate. J Urol. 1989;142:71-75.

3. Akduman B, Crawford ED. Transrectal ultrasound- guided prostate biopsy: current approach. Turkish Journal of Urology . 2010; 36(1): 25-32.

4. Autorino R, De Sio M, Di Lorenzo G, et al. How to decrease pain during transrectal ultrasound guided prostate biopsy: a look at the literature. J Urol. 2005;174:2091-2097

5. Soloway MS, Obek C. Periprostatic local anesthesia before ultrasound prostate biopsy. J Urol. 2000;163:172-173.

6. Skriapas K, Konstantinidis C, Samarinas M, et al. Comparison between lidocaine and glyceryl trinitrate ointment for perianal- intrarectal local anesthesia before transrectal ultrasonographyguided prostate biopsy: A placebo controlled trial. Urology. 2011; 77: 905-909.

7. Issa MM, Bux S, Chun $\mathrm{T}$, et al. A randomized prospective trial of intrarectal lidocaine for pain control during transrectal prostate biopsy: the Emory University experience. J Urol. 2000;164:397399.

8. Obek C, Ozkan B, Tunc B, et al. Comparison of 3 different methods of anesthesia before transrectal prostate biopsy: a prospective randomized trial. J Urol. 2004; 172:502-505.

9. Giannarini G, Autorino R, Valent F, et al. Combination of perianal-intrarectal lidocaine- prilocaine cream and periprostatic nerve block for pain control during transrectal ultrasound guided prostate biopsy: a randomized controlled trial. J Urol. 2009; 181: 585-91.

10. Naughton CK, Miller DC, Yan, Y, et al. Impact of transrectal ultrasound guided prostate biopsy on quality of life: a prospective randomized trial comparing 6 versus 12 cores. J Urol. 2001; 165: 100-3.

11. Rochester MA, LE Monnier K, Brewster SF, et al. A double-blind, randomized, controlled trial of topical glyceryl trinitrate for transrectal ultrasound guided prostate biopsy. J Urol. 2005;173:418-420.

12. McCabe JE, Hanchanale VS, Philip J, et al. A randomized controlled trial of topical glyceryl trinitrate before transrectal ultrasonographyguided biopsy of the prostate. BJU Int. 2007;100:536538.

13. Nash PA, Bruce JE, Indudhara R, et al. Transrectal ultrasound guided prostate nerve blockade eases systematic needle biopsy of the prostate. J Urol. 1996;155:607-609.

14. Stirling BN, Shockley KF, Carothers GG, et al. Comparison of local anesthesia techniques during transrectal ultrasound-guided prostate biopsies. Urology. 2002;60:89-92.

15. Raber M, Scattoni V, Roscigno M, et al. Perianal and intrarectal anaesthesia for transrectal biopsy of the prostate: a prospective randomized study comparing lidocaine- Prilocaine cream and placebo. BJU Int. 2005;96:1264-1267.

16. Alavi AS, Soloway MS, Vaidya A, et al. Local anesthesia for ultrasound guided prostate biopsy: a prospective randomized trial comparing 2 methods. J Urol. 2001;166:1343-1345.

17. Skriapas K, Konstandinidis C, Samarinas M, et al. Pain level and anal discomfort during transrectal ultrasound for guided prostate biopsy. Does intrarectal administration of local anesthetic before periprostatic anesthesia makes any difference? Minerva Urol Nefrol. 2009;61:137-142.

18. Jonas M, Amin S, Wright JW, et al. Topical 0.2 percent Glyceryl trinitrate ointment has a shortlived effect on resting anal pressure. Dis Colon Rectum. 2001;44:1640-1643. 